\title{
Malaria and risk of lymphoid neoplasms and other cancer: a nationwide population-based cohort study
}

Katja Wyss ${ }^{1,2^{*}}$, Fredrik Granath ${ }^{3}$, Andreas Wångdahl ${ }^{1,4}$, Therese Djärv ${ }^{5,6}$, Michael Fored $^{3}$, Pontus Naucler ${ }^{1,2}$ and Anna Färnert ${ }^{1,2}$

\begin{abstract}
Background: Malaria is associated with Burkitt lymphoma among children in Sub-Saharan Africa. No longitudinal studies have assessed the long-term risk of other lymphoma or cancer overall. Here, we investigated the risk of lymphoid neoplasms and other cancer after malaria.

Methods: We included 4125 patients diagnosed with malaria in Sweden in 1987-2015, identified either through the National Surveillance Database at the Public Health Agency of Sweden, the National Inpatient and Outpatient Register, or by reports from microbiology departments. A comparator cohort $(N=66,997)$ matched on sex, age and birth region was retrieved from the general population and an additional cohort with all individuals born in SubSaharan Africa registered in the Total Population Register in 1987-2015 ( $N=171,756)$. Incident lymphomas and other cancers were identified through linkage with the Swedish Cancer Register. Hazard ratios (HRs) were assessed using Cox regression with attained age as the timescale.

Results: A total of 20 lymphoid neoplasms and 202 non-haematological cancers were identified among malaria patients during a mean follow-up of 13.3 and 13.7 years, respectively. The overall risk of lymphoid neoplasms was not significantly increased (hazard ratio [HR] 1.24, 95\% confidence interval [CI] 0.79-1.94), neither did we find any association with all-site non-haematological cancer (HR 0.89,95\% Cl 0.77-1.02). However, in the Sub-Saharan Africa cohort, we observed an increased risk of lymphoid neoplasms after malaria diagnosis ( $H R 2.39,95 \% \mathrm{Cl} 1.06-5.40$ ), but no difference in the risk of other cancer ( $\mathrm{HR} 1.01,95 \% \mathrm{Cl} 0.70-1.45$ ). The association could not be explained by co-infection with HIV or chronic hepatitis B or C, since the risk estimate was largely unchanged after excluding patients with these comorbidities (HR 2.63,95\% Cl 1.08-6.42). The risk became more pronounced when restricting analyses to only including non-Hodgkin and Hodgkin lymphomas (HR 3.49,95\% Cl 1.42-8.56).

Conclusion: Individuals born in malaria-endemic areas and diagnosed with malaria in Sweden had an increased risk of lymphoid neoplasms, especially B cell lymphoma. There was no association with cancer overall nor did single malaria episodes confer an increased risk in travellers.
\end{abstract}

Keywords: Malaria, Lymphoid neoplasms, Lymphoma, Cancer

\footnotetext{
* Correspondence: katja.wyss@ki.se

'Division of Infectious Diseases, Department of Medicine Solna, Karolinska Institutet, Stockholm, Sweden

${ }^{2}$ Department of Infectious Diseases, Karolinska University Hospital, 17176 Stockholm, Sweden

Full list of author information is available at the end of the article
}

(C) The Author(s). 2020, corrected publication December 2020. Open Access This article is licensed under a Creative Commons Attribution 4.0 International License, which permits use, sharing, adaptation, distribution and reproduction in any medium or format, as long as you give appropriate credit to the original author(s) and the source, provide a link to the Creative Commons licence, and indicate if changes were made. The images or other third party material in this article are included in the article's Creative Commons licence, unless indicated otherwise in a credit line to the material. If material is not included in the article's Creative Commons licence and your intended use is not permitted by statutory regulation or exceeds the permitted use, you will need to obtain permission directly from the copyright holder. To view a copy of this licence, visit http://creativecommons.org/licenses/by/4.0/. The Creative Commons Public Domain Dedication waiver (http:// creativecommons.org/publicdomain/zero/1.0/) applies to the data made available in this article, unless otherwise stated in a credit line to the data. 


\section{Background}

Nearly half of the world's population lives at risk of malaria [1]. The acute complications of malaria are well known, but in addition to direct malaria-related morbidity and mortality, there is increasing evidence that malaria has several long-term effects. One such consequence is endemic Burkitt lymphoma, the most common cancer in children in Sub-Saharan Africa [2, 3]. The International Agency for Research on Cancer (IARC) has declared Plasmodium falciparum as a probable carcinogenic agent in humans [4], but besides the well-established association with Burkitt lymphoma [5-7], epidemiological evidence is limited.

Occasional epidemiological reports from endemic areas have suggested a link between malaria and non-Burkitt lymphomas in adults [8-10], as well as cervical cancer in women living in high-endemic regions of Uganda [11]. A few case-control studies from non- or low-endemic countries indicate that malaria exposure could be associated with the non-endemic (sporadic) form of Burkitt lymphoma diagnosed at adult age [12], as well as non-Burkitt non-Hodgkin lymphomas [13, 14], lymphatic malignancies [15] and nasopharyngeal carcinoma in South East Asia [16]. Most of these studies are based on self-reported history of malaria, thus prone to exposure misclassification. One nested-case control study from the UK used longitudinally collected data from primary care records to assess previous malaria exposure [12], but otherwise, there are to our knowledge no publications with longitudinal design assessing the risk of lymphomas or other cancers in adults with previous malaria. Such investigations are difficult to manage in endemic areas where reliable health and recording systems often are lacking and the degree of malaria exposure is complicated to determine due to repeated and asymptomatic infections. Here, we have taken advantage of the unique health and population registries in Sweden to study long-term effects of malaria diagnosed in a mixed population of travellers and migrants.

We performed a nationwide population-based cohort study of patients diagnosed with malaria in Sweden, with the aim to investigate if malaria is associated with an increased risk of lymphoid neoplasms and/or other cancer.

\section{Methods}

\section{Setting}

Residents in Sweden are issued a unique personal identification number (PIN) that is recorded in all health and census registers, enabling register linkage [17]. Malaria is a notifiable disease under the Communicable Diseases Act, and microbiologically confirmed cases (by microscopy of thick and thin blood films or, infrequently, by polymerase chain reaction) should be reported to the Public Health Agency of Sweden both by the treating clinician and the diagnosing microbiology laboratory.
Malaria is generally managed by infectious disease specialists at larger hospitals, although other departments are involved in the treatment of children, pregnant women or complications requiring other specialists. Most patients diagnosed with malaria are admitted, but semi-immune individuals with mild symptoms are sometimes managed on an outpatient basis.

The Swedish National Patient Register (NPR) at the National Board of Health and Welfare includes diagnoses for an estimated 99\% of all hospitalizations in Sweden, with nationwide coverage since 1987 [18]. Since 2001, specialised outpatient care also is included. The Total Population Register (TPR) at Statistics Sweden contains demographic data for all inhabitants with PIN from 1969, including sex, date and country of birth, date of death and dates and countries of immigration and emigration.

\section{Study population \\ Exposed cohort}

Malaria patients were identified through four different sources (Fig. 1a):

1. All notified cases of malaria with complete PIN reported in the National Surveillance Database at the Public Health Agency of Sweden 1987-2015 ( $N=2884$ unique patients)

2. Additional patients with microbiologically confirmed malaria identified through hospital or laboratory records from infectious disease or microbiology departments $(N=39)$

3. Additional patients discharged with a diagnosis of malaria, according to the International Classification of Disease ICD-10: B50.0-B54.9 or ICD-9, 084A-H, 084W and 084X, in the National Inpatient Register 1987-2015 ( $N=949)$.

4. Additional patients with a diagnosis of malaria (same ICD codes as above) in the National Outpatient Register 2001-2015 ( $N=787)$.

Patients from sources 1 and 2 were considered confirmed whereas cases identified via sources 3 and 4 were not necessarily microbiologically verified. For confirmed malaria diagnosed in 1995-2015, data was enriched with clinical variables from medical records, such as disease severity and Plasmodium species, used in previous studies [19-21].

To increase the specificity of malaria identified via the NPR, we only included the main diagnoses of malaria from the infectious diseases, paediatric and emergency medicine departments in the Outpatient Register $(N=$ 344 excluded), while all patients discharged with a diagnosis of malaria were included from the Inpatient Register except those with secondary diagnoses from the departments other than infectious diseases, paediatrics, 


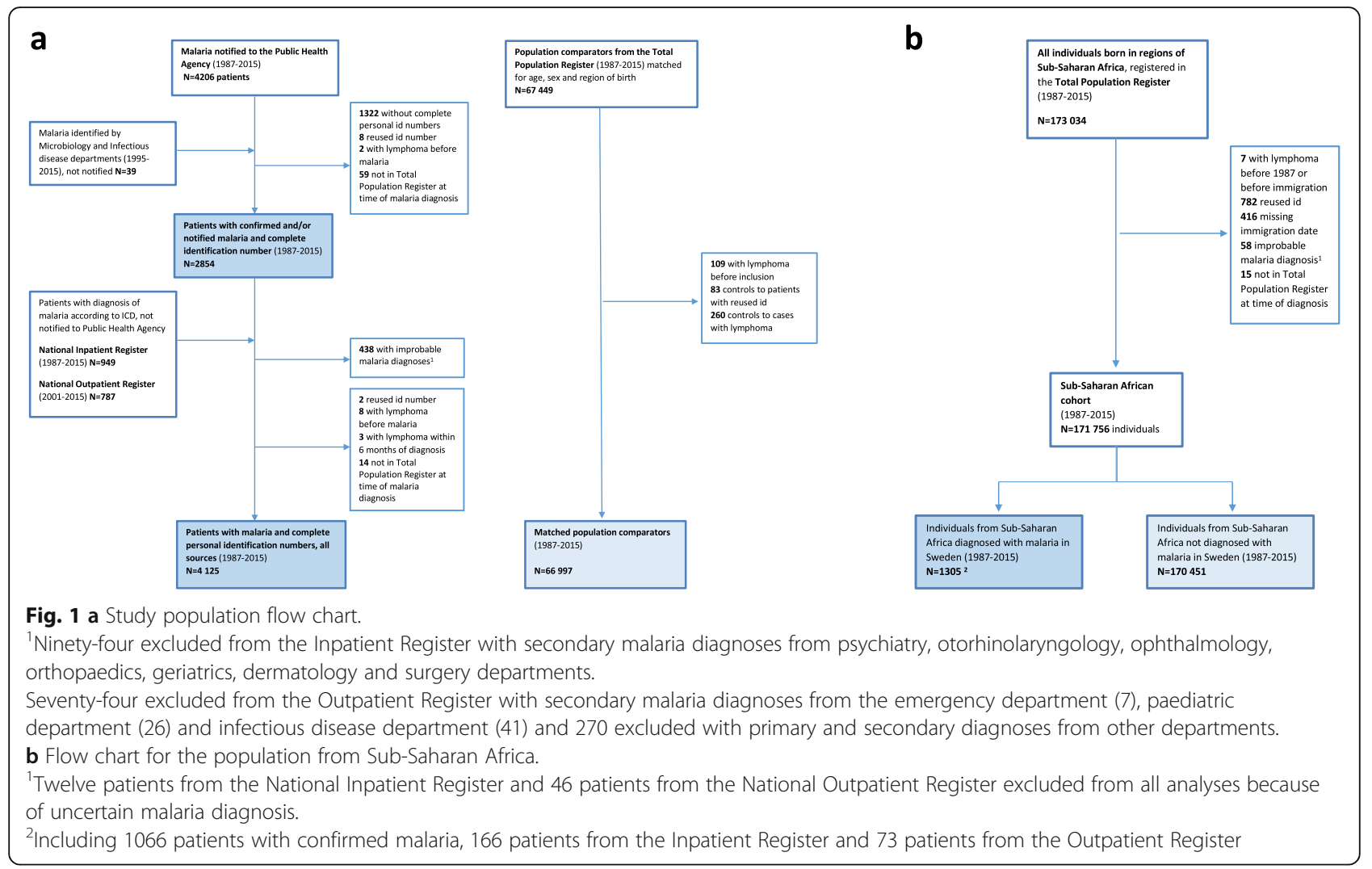

intensive care, internal medicine and obstetrics/gynaecology, and no follow-up at an infectious disease department $(N=94$ excluded). For individuals with multiple episodes, inclusion was set at the date of the first episode with confirmed malaria.

A total of 186 patients were not registered in the TPR at the time of malaria diagnosis; however, of these, $113 \mathrm{had}$ an immigration date within 1 year after diagnosis and were included from this date. Often, the actual date of immigration has occurred earlier but is set to the date of registration in Sweden (personal communication with Jonas Färnstrand, Statistics Sweden). Before becoming registered in Sweden, immigrants in contact with the health care system obtain a temporary identification number that can be connected to the PIN via electronic patient records, thereby enabling inclusion as a malaria case.

Depending on the outcome analysed, patients with either prior diagnosis of lymphoid neoplasm or all-site cancer were excluded. To minimise the possibility of misclassifying patients identified by ICD-codes in the NPR, lymphoid neoplasms and cancers diagnosed within 6 months after the malaria diagnosis were also excluded.

\section{Comparator cohort}

For each malaria patient, we randomly selected 20 comparators from the TPR, matched for sex, birth year $( \pm 1$ year) and region of birth categorised according to the
United Nations geoscheme [22], alive and without previous malaria diagnosis on the date of malaria of the corresponding patient. Individuals with previous lymphoma or cancer were excluded.

Based on a previous study of imported malaria in Sweden, approximately $50 \%$ of patients with confirmed malaria had immigrated from countries where malaria is highly endemic [21]. In order to better control for host factors including malaria exposure and immunity, an additional cohort was assembled from the TPR composed of all individuals born in Sub-Saharan Africa, defined as the United Nations geoscheme regions Eastern Africa, Western Africa, Middle Africa and Southern Africa, who were alive and registered in Sweden at some point between 1987 and 2015 (Fig. 1b). We refer to these regions as "endemic" and all other regions as "low- or non-endemic".

\section{Register linkage and follow-up}

The study population was followed from the inclusion date, i.e. first episode of malaria or corresponding date for comparators, to the first diagnosis of lymphoma or cancer, emigration, death or end of study (31 January 2016), whichever occurred first. For unexposed individuals, follow-up also ended if malaria was diagnosed, and the comparator then became included as exposed. Follow-up was achieved through linkage to the following registers: 
1. The Cancer Register at National Board of Health and Welfare includes the date and type of all incident cancers diagnosed in Sweden from 1958. Reporting is mandatory by both clinician and pathologist and completeness estimated to $>96$ [23].

2. The TPR at Statistics of Sweden includes date and country of birth as well as migration data.

3. Cause of Death Register at National Board of Health and Welfare includes date and causes of death.

The cohort from Sub-Saharan Africa was followed from the first immigration date to Sweden, or from 1 January 1987 if immigration occurred before 1987. Individuals were censored at emigration but were allowed to re-enter the study in case of subsequent immigration episodes; thus, all registered periods of residency in Sweden were included.

\section{Outcome}

Outcomes were defined as the first diagnosis of lymphoid neoplasms (ICD-7: 200-204) or the first diagnosis of all-site cancer excluding haematological malignancies and non-melanoma skin cancers (ICD-7: 140-190,192199). In a subanalysis, we also assessed the risk of malignant lymphoma (non-Hodgkin, NHL and Hodgkin) using a stricter definition excluding chronic lymphatic leukaemia (CLL), myeloma, acute lymphoblastic leukaemia (ALL) and lymphoproliferative disorders. For this purpose, histopathology diagnoses referred to as SNOMED codes (Systematized Nomenclature of Medicine), available from 1993, were used for a more precise classification of the different types of lymphoid neoplasms as well as subclassification of NHL, according to InterLymph [24, 25].

\section{Statistics}

We used Stata version 14 (StataCorp) for statistical analyses and SAS version 9.4 for data cleaning. Crude incidence rates and 95\% confidence intervals (CIs) were calculated as the number of events (first diagnosis of lymphoid neoplasms, or all site non-haematological cancer during follow-up) per 1000 person-years. We estimated relative risks for malaria cases compared to the comparator cohort as hazard ratios (HR) with 95\% CIs using Cox proportional hazard regression, stratified for sex, patient birth region (Sub-Saharan Africa vs non/ low-endemic regions), calendar period for study entry (1987 to 1994, 1995 to 2004, 2005 to 2015) and followup time $(<5,5$ to 9,10 to 19 and 20 to 30 years. Attained age was used as underlying timescale throughout the study; thus, all analyses were adjusted for age. Additional adjustments were made for sex, birth region and calendar period. The proportional hazard assumption was assessed using Schoenfeldt residuals.
Assuming that comparators born in Sub-Saharan Africa to some extent also could have been malaria exposed before arriving in Sweden, we categorised the study population into four levels of assumed increasing malaria exposure: controls with non/low-endemic origin, malaria cases with non/low-endemic origin, controls with endemic origin and cases with endemic origin, and estimated HR for lymphoma and cancer overall for all categories compared to the group of expected lowest exposure (comparator subjects with non/low-endemic origin).

For the cohort of individuals born in Sub-Saharan Africa, malaria was treated as a time-varying covariate; thus, an individual diagnosed with malaria contributed with both unexposed and exposed time. Individuals were censored at emigration, but those that re-immigrated were included as new episodes from that date. The time in between emigration and immigration was not included in risk time. However, we created a variable for estimated total attained time spent in an endemic region, based on the country of re-immigration, as a measure of previous exposure. Crude incidence rates were calculated overall and separately for sex, calendar period, follow-up time, region of birth within Africa, age at first immigration $(<18,18$ to 29,30 to $39, \geq 40$ years), age at the end of follow-up ( 0 to 29,30 to 49 and $\geq 50$ years) and time in endemic region ( 0 to 9,10 to 19,20 to 29 and $\geq 30$ years). Crude and adjusted HRs were stratified for total attained time in endemic region and sex.

A restricted analysis was performed excluding individuals with a diagnosis of HIV, chronic hepatitis B or C identified in the NPR (ICD 10: B20-B24, Z21, B18.0B18.1, B18.2; ICD-9: 2795/279K, 079J, 0702-0705) before inclusion and censoring those diagnosed during follow-up at the date of HIV/hepatitis diagnosis, including up until 6 months after lymphoma diagnosis (assuming the chronic infection was present before lymphoma diagnosis).

\section{Results}

In total, 4125 individuals diagnosed with malaria in Sweden were included in the study. Among these, 2854 patients had microbiologically confirmed malaria, of which 2815 (98.6\%) notified to the Swedish Public Health Agency and 2009 (70.4\%) with available clinical data. Additionally, 1271 patients with unnotified malaria were identified through malaria ICD 9/10 codes from the Inpatients Register $(N=841)$ or Outpatient Register $(N=430)$. As it was not possible to find 20 controls for all patients born outside Sweden, the total number of controls was 66,997 (mean 16 controls per patient) (Fig. 1a). This explains the difference in the proportion of patients with origin in Sub-Saharan Africa, presented among other demographic characteristics in Table 1. Other differences noted were the higher proportion of HIV and hepatitis B diagnoses among malaria cases. 
Table 1 Characteristics of malaria patients and matched population comparators

\begin{tabular}{|c|c|c|}
\hline & $\begin{array}{l}\text { Malaria patients, all sources }{ }^{\mathrm{a}} \\
(N=4125)^{\mathrm{b}}\end{array}$ & $\begin{array}{l}\text { Matched comparator cohort from the general } \\
\text { population }(N=66,997)\end{array}$ \\
\hline Sex, female, $n(\%)$ & $1622(39.3)$ & $26,991(40.3)$ \\
\hline \multicolumn{3}{|l|}{ Age } \\
\hline Age at start of follow-up, mean ( \pm SD) years & $34.7( \pm 18.5)$ & $35.5( \pm 18.9)$ \\
\hline Attained age at end of follow-up, mean ( \pm SD) years & $48.0( \pm 18.7)$ & $49.6( \pm 19.0)$ \\
\hline Children $<18 \mathrm{y}$, at inclusion, $n(\%)$ & $676(16.4)$ & $9627(14.4)$ \\
\hline Age $\geq 65 \mathrm{y}$, at inclusion, $n(\%)$ & $273(6.6)$ & $5091(7.6)$ \\
\hline \multicolumn{3}{|l|}{ Follow-up } \\
\hline Total follow-up, person-years & 54,739 & 943,828 \\
\hline Average follow-up, person-years (SD) & $13.3( \pm 8.3)$ & $14.1( \pm 8.4)$ \\
\hline \multicolumn{3}{|l|}{ Country of birth ${ }^{c}$} \\
\hline Sub-Saharan Africa, $n$ (\%) & $1307(31.7)$ & $12,758(19.0)$ \\
\hline Other low-endemic regions, $n$ (\%) & $295(7.2)$ & $4899(7.3)$ \\
\hline Sweden/non-endemic region, $n(\%)$ & $2523(61.1)$ & $49,332(73.6)$ \\
\hline \multicolumn{3}{|l|}{ Co-infections } \\
\hline HIV before/at inclusion, or during follow-up ${ }^{d}, n(\%)$ & $54(1.3), 49(1.2)$ & $228(0.3), 164(0.2)^{3}$ \\
\hline Hepatitis B before/at inclusion, or during follow-up ${ }^{e}, n(\%)$ & $26(0.6), 39(1.0)$ & $235(0.4), 307(0.5)$ \\
\hline Hepatitis C before/at inclusion, or during follow-up, $n$ (\%) & $14(0.3), 19(0.5)$ & $124(0.2), 333(0.5)$ \\
\hline Incident lymphoid neoplasms, $n$ (\%) & $20(0.48)$ & $304(0.45)$ \\
\hline Lymphoid neoplasm within 1 year, $n(\%)$ & 0 & $17(5.6)$ \\
\hline Lymphoid neoplasm after 1-5 y, n (\%) & $5(25.0)$ & $65(21.4)$ \\
\hline \multirow[t]{2}{*}{ Lymphoid neoplasm after > 5 y, $n(\%)$} & $15(75.0)$ & $222(73.0)$ \\
\hline & $\begin{array}{l}\text { Malaria patients, all sources } \\
(N=4027)^{f}\end{array}$ & $\begin{array}{l}\text { Matched comparator cohort from the general } \\
\text { population }(N=63,588)\end{array}$ \\
\hline Incident cancer ${ }^{\mathrm{g}}, \mathrm{n}(\%)$ & $202(5.0)$ & $3993(6.2)$ \\
\hline Total follow-up, person-years & 55,361 & 924,517 \\
\hline Average follow-up, person-years (SD) & $13.7( \pm 8.1)$ & $14.5( \pm 8.3)$ \\
\hline
\end{tabular}

$N$ total number, $n$ number, $S D$ standard deviation, $y$ years

ancludes 2854 patients with confirmed malaria, of which 2815 patients notified to the Public Health Agency 1987-2015 and 39 (1.4\%) cases not notified to the Public Health Agency but microbiological confirmed. Additional 1271 patients with unnotified malaria identified through the National Inpatient Register 1987$2015(N=841)$ and Outpatient Register 2001-2015 ( $n=430)$ with a diagnosis of malaria according to ICD-10 (B50.0-B54.9) or ICD-9 (084A-H, 084W, 084X) ${ }^{b}$ Patients with previous lymphoid neoplasms excluded: 2 patients with notified malaria and 8 patients with malaria identified in the National Inpatient Register. Three malaria cases from the National Inpatient Register were excluded because of lymphoid neoplasm within 6 months

${ }^{c} n=8$ of comparators with missing information on country of birth. Two malaria cases with missing information on birth where data on birth country was retrieved through the clinical database

${ }^{\mathrm{d}}$ Additionally, 1 case of HIV after lymphoma in a malaria patient and 4 among comparators

${ }^{\mathrm{e} A d d i t i o n a l l y, ~} 1$ case of hepatitis B after lymphoma in a malaria patient

fPatients with previous cancer (non-haematological) were excluded: 98 malaria patients with previous cancer and 16 with cancer within 6 months

${ }^{9}$ All cancers except for non-melanoma skin cancers, leukaemia and lymphoma (ICD-7: 140-190, 192-199)

\section{Risk of lymphoid neoplasms}

The 4125 malaria patients were followed for 54,739 personyears, during which we observed 20 cases of lymphoid neoplasms, corresponding to a crude incidence of 0.37 per 1000 person-years (95\% CI 0.24-0.57). Three hundred four lymphoid neoplasms were observed among the matched comparators during 943,828 person-years, corresponding to a crude incidence of 0.32 per 1000 person-years (95\% CI $0.29-0.36)$. The majority $(15 / 20)$ of lymphoid neoplasms among the malaria cases were diagnosed more than 5 years after the malaria diagnosis (Table 1).
The overall risk for incident lymphoid neoplasms among malaria patients was not increased (HR 1.24, 95\% CI 0.791.94). There was a modest difference in the risk estimate of men and women with malaria, a tendency of higher risk in individuals born in Sub-Saharan Africa (HR 1.97, 95\% CI 0.81-4.81) and inclusion after 2005 (HR 2.29, 95\% CI 0.90-5.80). The overall risk was not affected by adjustment for sex, region of birth and calendar period for entry (adjusted HR [adjHR] 1.20, 95\% CI 0.76-1.88) and was similar in the analysis restricted to confirmed cases only (HR 1.26, 95\% CI 
0.73-2.16, adjHR 1.20, 95\% CI 0.69-2.07). However, for individuals of endemic origin, the association became somewhat more pronounced among confirmed cases (HR 2.32, 95\% CI 0.94-5.76) (Table 2).

By categorising patients into four levels of exposure based on birth region and malaria diagnosis, we observed that malaria patients of endemic origin had a higher risk of lymphoid neoplasms compared to the population from non/low-endemic regions without previous malaria diagnosis (HR 2.36, 95\% CI 1.05-5.33, adjHR 2.24, 95\% CI 0.99-5.07), also when restricting the analysis to confirmed cases only (HR 2.94, 95\% CI 1.296.67 and adjHR 2.88, 95\% CI 1.27-6.54), while the risk for non-haematological cancer was rather decreased (HR 0.60, 95\% CI 0.41-0.86, adjHR 0.61, 95\% CI 0.42-0.90) (Additional file 1: Table S1).

SNOMED codes were available for all the lymphoid neoplasms among malaria cases and 98\% of comparators. Histological classification showed that nonHodgkin lymphoma was the most common type of lymphoid neoplasm among both malaria patients (63\%) and in the comparator cohorts (72\%). Two $(10 \%)$ of the malaria patients with lymphoid neoplasms and $14(5 \%)$ of the comparators had Hodgkin. Chronic lymphocytic leukaemia (CLL), one of the most common haematological disorder seen among Swedish controls (19\%), was not as frequent among malaria patients $(10 \%)$, and no cases were seen among malaria patients with endemic origin (Additional file 1: Table S2). The only case of Burkitt lymphoma was in an adult of endemic origin with a confirmed malaria diagnosis. There were additional variations in the proportion of NHL subgroups depending on the exposure and origin, but numbers were too small for comparative analysis. With the stricter lymphoma definition, a total of 12 malignant lymphomas were identified among malaria patients and 164 among comparators, but the risk was largely unchanged (HR 1.36, 95\% CI 0.75-2.44).

In the subset of malaria patients with clinical data $(N=2009)$, we were able to assess disease severity, but none of the patients who later developed lymphoma had severe malaria according to WHO's definition (WHO 2015). Among confirmed malaria cases, we also determined multiple episodes (including only new infections, not relapses or recrudescence) and species. One hundred seventy-nine of 2854 cases had two episodes and 25/2854 three or more additional episodes. However, none of these patients developed lymphoma. Among the 14 patients with confirmed malaria who later developed lymphoid neoplasms, most had been infected with Plasmodium falciparum $(11 / 14)$, only one with Plasmodium vivax and two with Plasmodium ovale.

\section{Risk of other cancer}

During follow-up, 202 non-haematological cancers were observed among the malaria cases and 3933 among the comparators, corresponding to a crude incidence rate of 3.65 (95\% CI 3.18-4.19) cancers/1000 person-years among malaria patients and 4.25 (95\% CI 4.12-4.39)/ 1000 person-years among malaria-free comparators. There was no significant difference in the overall risk for incident cancer for malaria patients, rather a tendency of decreased risk (HR 0.89, 95\% CI 0.77-1.02), also after adjustment for sex, calendar period and birth region (adjHR 0.92, 95\% CI 0.80-1.06) and after restricting the analysis to confirmed cases only (HR 0.98, 95\% CI $0.83-$ 1.16, adjHR 1.02, 95\% CI 0.86-1.21) (Table 2).

We also investigated the distribution of cancer types according to malaria exposure and region of birth. The proportion of lymphoid neoplasms was markedly increased among malaria patients with Sub-Saharan origin (18\%) compared to both comparators with the same origin (9\%), as well as malaria patients (5\%) and controls (6\%) with non/low-endemic origin. Except for a high proportion of endocrinal tumours (mainly thyroid gland) and liver cancers among both malaria patients and comparators from Sub-Saharan Africa, no other major differences in the distribution of cancer types were observed (Additional file 1: Table S3).

\section{Sub-Saharan African cohort}

In the population born in Sub-Saharan Africa $(N=171$, 756 individuals with 179,147 registered periods in Sweden), there was a total of 212 lymphoid neoplasms, six of these in 15,497 exposed person-years in 1305 individuals diagnosed with malaria in Sweden and 206 lymphoid neoplasms during 1,720,550 unexposed person-years in 170,451 individuals without previous malaria diagnosis, conferring to an event rate of 0.39 (95\% CI $0.17-0.86)$ and 0.12 (95\% CI $0.10-0.14$ ), respectively (Table 3 ).

The risk of developing a lymphoid neoplasm was more than twice as high (HR 2.39, 95\% CI 1.06-5.40) after a diagnosis of malaria, also after adjusting for sex and calendar period (adjHR 2.33, 95\% CI 1.03-5.26) (Table 4). And when restricting the analysis to confirmed cases only, the association became stronger (HR 2.97, 95\% CI 1.32-6.70, adjHR 2.88, 95\% CI 1.27-6.51) (Table 4).

A tendency of stronger association among individuals with long attained time ( $\geq 30$ years) in the endemic country as well as female sex was observed in stratified analyses (Table 4), but no interaction with neither total attained time (HR 3.18 for malaria diagnosis and time $\geq 30$ years, 95\% CI 1.01-10.03, $p=0.49$ ) nor gender (HR 3.21 for malaria diagnosis and female sex, 95\% CI 0.79 13.06, $p=0.10$ ) could be demonstrated except for $\geq 30$ 
Table 2 Hazard ratios for incident lymphoid neoplasms and other cancer in malaria patients

\begin{tabular}{|c|c|c|c|c|c|c|}
\hline & \multicolumn{3}{|c|}{ Malaria, all sources ${ }^{a}(N=4125)$} & \multicolumn{3}{|c|}{ Confirmed malariab $^{\mathrm{b}}(N=2854)$} \\
\hline & $\begin{array}{l}\text { Lymphoid neoplasms } \\
\text { among malaria patients, } \\
n(\%)\end{array}$ & $\begin{array}{l}\text { Lymphoid neoplasms } \\
\text { among comparators, } \\
n(\%)\end{array}$ & $\mathrm{HR}(95 \% \mathrm{Cl})$ & $\begin{array}{l}\text { Lymphoid } \\
\text { neoplasms among } \\
\text { malaria patients, } \\
n(\%)\end{array}$ & $\begin{array}{l}\text { Lymphoid } \\
\text { neoplasms } \\
\text { among } \\
\text { comparators, } \\
n(\%)\end{array}$ & $\mathrm{HR}(95 \% \mathrm{Cl})$ \\
\hline All & 20 & 304 & $1.24(0.79-1.94)$ & 14 & 194 & $1.26(0.73-2.16)$ \\
\hline \multicolumn{7}{|l|}{ Sex } \\
\hline Male & $13(65.0)$ & $210(69.1)$ & $1.12(0.64-1.95)$ & $9(64.2)$ & $142(73.2)$ & $1.09(0.56-2.14)$ \\
\hline Female & $7(35.0)$ & $94(30.9)$ & $1.49(0.69-3.20)$ & $5(35.7)$ & $52(26.8)$ & $1.68(0.67-4.22)$ \\
\hline \multicolumn{7}{|l|}{ Follow-up time (y) } \\
\hline$<5$ & $5(25.0)$ & $82(27.0)$ & $0.91(0.37-2.24)$ & $2(14.3)$ & $45(23.2)$ & $0.64(0.16-2.65)$ \\
\hline $5-9$ & $5(25.0)$ & $78(25.7)$ & $1.06(0.43-2.62)$ & $4(28.6)$ & $48(24.7)$ & $1.30(0.57-3.60)$ \\
\hline $10-19$ & $7(35.0)$ & $108(35.5)$ & $1.24(0.58-2.67)$ & $7(50.0)$ & $80(41.2)$ & $1.45(0.67-3.14)$ \\
\hline $20-30$ & $3(15.0)$ & $36(11.8)$ & $1.38(0.42-4.51)$ & $1(7.1)$ & $21(10.8)$ & $0.86(0.12-6.43)$ \\
\hline \multicolumn{7}{|l|}{ Years of entry } \\
\hline 1987-1994 & $9(45.0)$ & 109 (35.9) & $1.51(0.77-2.99)$ & $6(42.9)$ & $67(34.5)$ & $1.69(0.73-3.90)$ \\
\hline 1995-2004 & $6(30.0)$ & $155(51.0)$ & $0.73(0.32-1.66)$ & $5(35.7)$ & $103(53.1)$ & $0.81(0.33-2.00)$ \\
\hline $2005-2015$ & $5(25.0)$ & $40(13.2)$ & $2.29(0.90-5.80)$ & $3(21.4)$ & $24(12.4)$ & $1.90(0.57-6.33)$ \\
\hline \multicolumn{7}{|l|}{ Region of birth } \\
\hline Sub-Saharan Africa & $6(30.0)$ & $25(8.2)$ & $1.97(0.81-4.81)$ & $6(42.9)$ & $21(10.8)$ & $2.32(0.94-5.76)$ \\
\hline \multirow[t]{3}{*}{ Non/low-endemic } & $14(70.0)$ & $279(91.8)$ & $1.05(0.61-1.80)$ & $8(57.1)$ & $173(89.2)$ & $0.91(0.45-1.85)$ \\
\hline & \multicolumn{3}{|c|}{ Malaria, all sources ${ }^{c}(N=4027)$} & \multicolumn{3}{|c|}{ Confirmed malaria $^{d}(N=2812)$} \\
\hline & $\begin{array}{l}\text { Cancer among } \\
\text { malaria patients, } \\
n(\%)\end{array}$ & $\begin{array}{l}\text { Cancer among } \\
\text { comparators, } \\
n(\%)\end{array}$ & $\mathrm{HR}(95 \% \mathrm{Cl})$ & $\begin{array}{l}\text { Cancer }{ }^{\mathrm{e}} \text { among } \\
\text { malaria patients, } \\
n(\%)\end{array}$ & $\begin{array}{l}\text { Cancer among } \\
\text { comparators, } \\
n(\%)\end{array}$ & $\mathrm{HR}(95 \% \mathrm{Cl})$ \\
\hline All & 202 & 3933 & $0.89(0.77-1.02)$ & 145 & 2559 & $0.98(0.83-1.16)$ \\
\hline \multicolumn{7}{|l|}{ Sex } \\
\hline Male & $112(55.5)$ & $2245(57.1)$ & $0.89(0.74-1.07)$ & $85(58.6)$ & $1522(59.5)$ & $1.00(0.80-1.24)$ \\
\hline Female & $90(44.6)$ & $1688(42.9)$ & $0.89(0.72-1.10)$ & $60(41.4)$ & $1037(40.5)$ & $0.94(0.73-1.23)$ \\
\hline \multicolumn{7}{|l|}{ Follow-up time (y) } \\
\hline$<5$ & $44(21.8)$ & $947(24.1)$ & $0.72(0.53-0.98)$ & $27(18.6)$ & 498 (19.5) & $0.70(0.48-1.04)$ \\
\hline $5-9$ & $50(24.8)$ & $944(24.0)$ & $0.77(0.58-1.03)$ & $36(24.8)$ & 605 (23.6) & $0.82(0.59-1.15)$ \\
\hline $10-19$ & $81(40.1)$ & $1498(38.1)$ & $0.86(0.69-1.08)$ & $60(41.4)$ & $1077(42.1)$ & $0.86(0.67-1.12)$ \\
\hline $20-30$ & $27(13.4)$ & $544(13.8)$ & $0.96(0.65-1.41)$ & $22(15.2)$ & 379 (14.8) & $1.17(0.76-1.79)$ \\
\hline \multicolumn{7}{|l|}{ Years of entry } \\
\hline 1987-1994 & 79 (39.1) & $1510(38.4)$ & $0.99(0.79-1.24)$ & $58(40.0)$ & 985 (38.5) & $1.16(0.89-1.51)$ \\
\hline 1995-2004 & $101(50.0)$ & $1939(49.3)$ & $0.86(0.70-1.05)$ & $77(53.1)$ & $1331(52.0)$ & $0.94(0.74-1.18)$ \\
\hline 2005-2015 & $22(10.1)$ & $484(12.3)$ & $0.72(0.47-1.11)$ & $10(6.9)$ & $243(9.5)$ & $0.62(0.33-1.16)$ \\
\hline \multicolumn{7}{|l|}{ Region of birth } \\
\hline Sub-Saharan Africa & $26(12.9)$ & $207(5.3)$ & $0.99(0.66-1.50)$ & $19(13.1)$ & $160(6.3)$ & $0.93(0.58-1.49)$ \\
\hline Non/low-endemic & $176(87.1)$ & $3726(94.7)$ & $0.92(0.79-1.07)$ & $126(86.9)$ & $2399(93.8)$ & $1.05(0.87-1.25)$ \\
\hline
\end{tabular}

$C l$ confidence interval, $H R$ hazard ratio, $N$ total number, $n$ number, $y$ years

aPatients with malaria identified by all sources (Public Health Agency, microbiology and infectious disease departments, National Patient Register), without previous lymphoid neoplasms

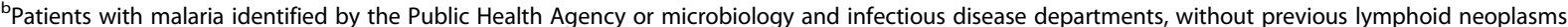
'Patients with malaria identified by all sources (Public Health Agency, microbiology and infectious disease departments, National Patient Register), without previous cancer

${ }^{\mathrm{d} P a t i e n t s}$ with malaria identified by the Public Health Agency or microbiology and infectious disease departments, without previous cancer

${ }^{\mathrm{e} A l l}$ cancers except for non-melanoma skin cancers, leukaemia and lymphoma (ICD-7: 140-190, 192-199) 
years in confirmed cases of malaria (HR 3.18, 95\% CI $1.01-10.03, p=0.049$ ).

After excluding individuals with a diagnosis of HIV or chronic hepatitis $\mathrm{B}$ or $\mathrm{C}$ before inclusion and censoring those diagnosed during follow-up, a total of 5 lymphoid neoplasms were observed among malaria patients and 154 among comparators. The overall risk for lymphoid neoplasms after malaria was still increased (HR 2.63, 95\% CI 1.08-6.42), also after adjustments for sex and calendar period (HR 2.50, 95\% CI 1.02-6.11) (Additional file 1: Table S4).

The risk for all-site non-haematological cancer did not differ significantly for malaria exposed vs unexposed (HR 1.01, 95\% CI 0.70-1.45), independently of sex and calendar period (HR 1.14, 95\% CI 0.79-1.63) (Table 4).

The malaria patients that developed lymphoid neoplasms all had confirmed malaria, were born in middle or western Africa, had immigrated to Sweden at 28-34 years of age and developed lymphoma after at least 10 years in Sweden. Four patients were diagnosed with lymphoma 5 to 13 years after the malaria diagnosis and the other two between 1 and 5 years after. Except one Hodgkin lymphoma and one myeloma, the remaining four (67\%) were B cell-derived non-Hodgkin lymphoma, one of these a Burkitt lymphoma. Among the Sub-Saharan population without previous malaria diagnosed in Sweden, 119 (58\%) of all lymphoid neoplasms were NHL (Additional file 1: Table S2). We observed a more pronounced risk in malaria patients when using the stricter definition of only malignant lymphoma (HR 3.49 , 95\% CI $1.42-$ 8.56, adjHR 3.27, 95\% CI 1.33-8.04), also when restricting the analysis to confirmed cases (HR 4.35, 95\% CI 1.77-10.67, adjHR 4.05, 95\% CI 1.65-9.96).

Among individuals without malaria diagnosis, $16 \%$ of those who developed a lymphoid neoplasm were children or young adults ( $<30$ years) at the time of diagnosis, and most lymphoid neoplasms were diagnosed at 30-49 years of age. Among malaria patients, the majority were diagnosed with lymphoma at $>50$ years of age, and no one was diagnosed $<30$ years of age (Table 3 ).

\section{Discussion}

In this nationwide population-based cohort study of patients diagnosed with malaria in Sweden, we observed an over two times increased risk of incident lymphoid neoplasms in patients born in Sub-Saharan Africa, but no increased risk for patients with malaria born in Sweden. The risk was more pronounced for B cell lymphoma and in confirmed cases of malaria. No risk of nonhaematological cancer was observed in malaria patients overall, and in patients from Sub-Saharan Africa, the risk was rather decreased.

\section{Comparisons with other studies}

Previous studies from endemic areas have established an association between holoendemic P. falciparum and Burkitt lymphoma in African children, and a possible link with non-Burkitt lymphomas and cervical cancers in adults, but have been based on regional differences in malaria transmission and cancer incidence $[6-8,11]$, sero-epidemiological comparisons involving anti-malaria antibodies [26, 27] or Mendelian randomisation approach of sickle cell traits [28]. A nested case-control study from the UK found an association with previous malaria exposure in adults with sporadic Burkitt lymphoma; however, the definition of exposure included both treatment for malaria, prescribed chemoprophylaxis and a self-reported history of malaria, thus not microbiologically confirmed [12]. The few studies from nonendemic countries, suggesting an association between malaria and other lymphoid malignancies, lack longitudinal design and are prone to recall bias [13-15].

Here, we followed patients diagnosed with malaria for up to 30 years and found that individuals born in SubSaharan Africa had an increased risk of developing lymphoid neoplasms, 1.5-13 years after a confirmed malaria diagnosis. The long time frame makes reverse causation, i.e. higher probability of symptomatic malaria in individuals with an undiscovered lymphoma, unlikely. The risk was most pronounced for lymphomas discovered at $>50$ years of age, indicating a prolonged effect and possibly different mechanisms compared to the endemic Burkitt lymphoma diagnosed in Sub-Saharan Africa which has a peak incidence at 6 years of age [29]. The only Burkitt lymphoma observed in our exposed population was a 52-year-old individual of endemic origin with confirmed P. falciparum and no known HIV or hepatitis.

\section{Interpretation}

Infections play an important role in many cancers, attributing to at least $20 \%$ of all cancers globally [30]. Lymphoid neoplasms form a heterogenetic group both regarding morphology and aetiology. However, in particular, B cell-derived lymphomas have been linked to chronic inflammation and infections [31].

While the oncogenic role of Epstein-Barr virus (EBV) has been extensively studied, the mechanism linking $P$. falciparum to lymphoma development is still not completely understood. Recent studies have proposed that repeated malaria infections in early childhood leads to expansion and reactivation of latently EBV-infected B cells and deregulated expression of AID (activation-induced cytidine deaminase), inducing DNA damage enabling the $c-m y c$ translocation characterising Burkitt lymphoma [32, 33]. In murine models, infections with Plasmodium species have been observed to increase the 


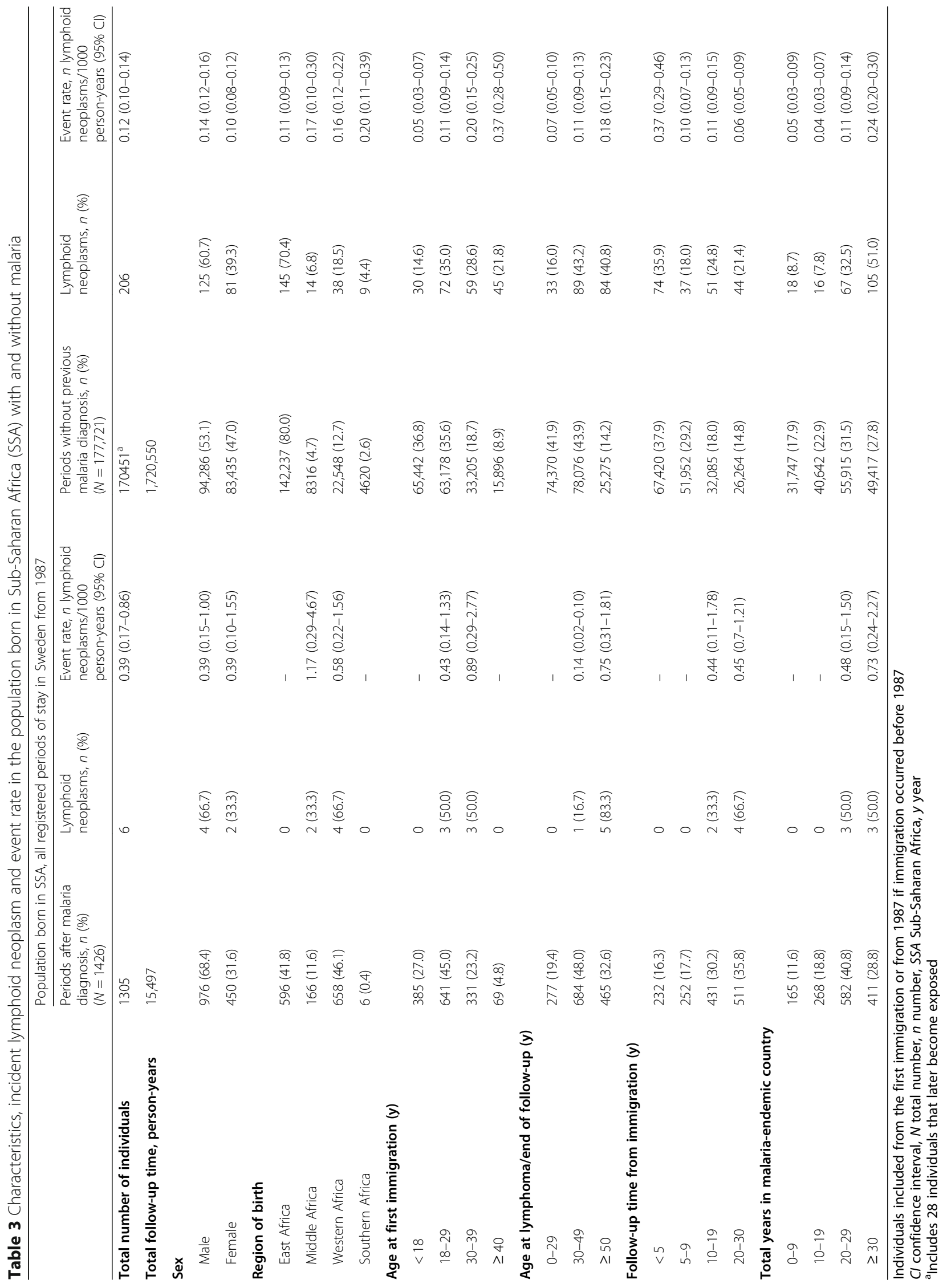




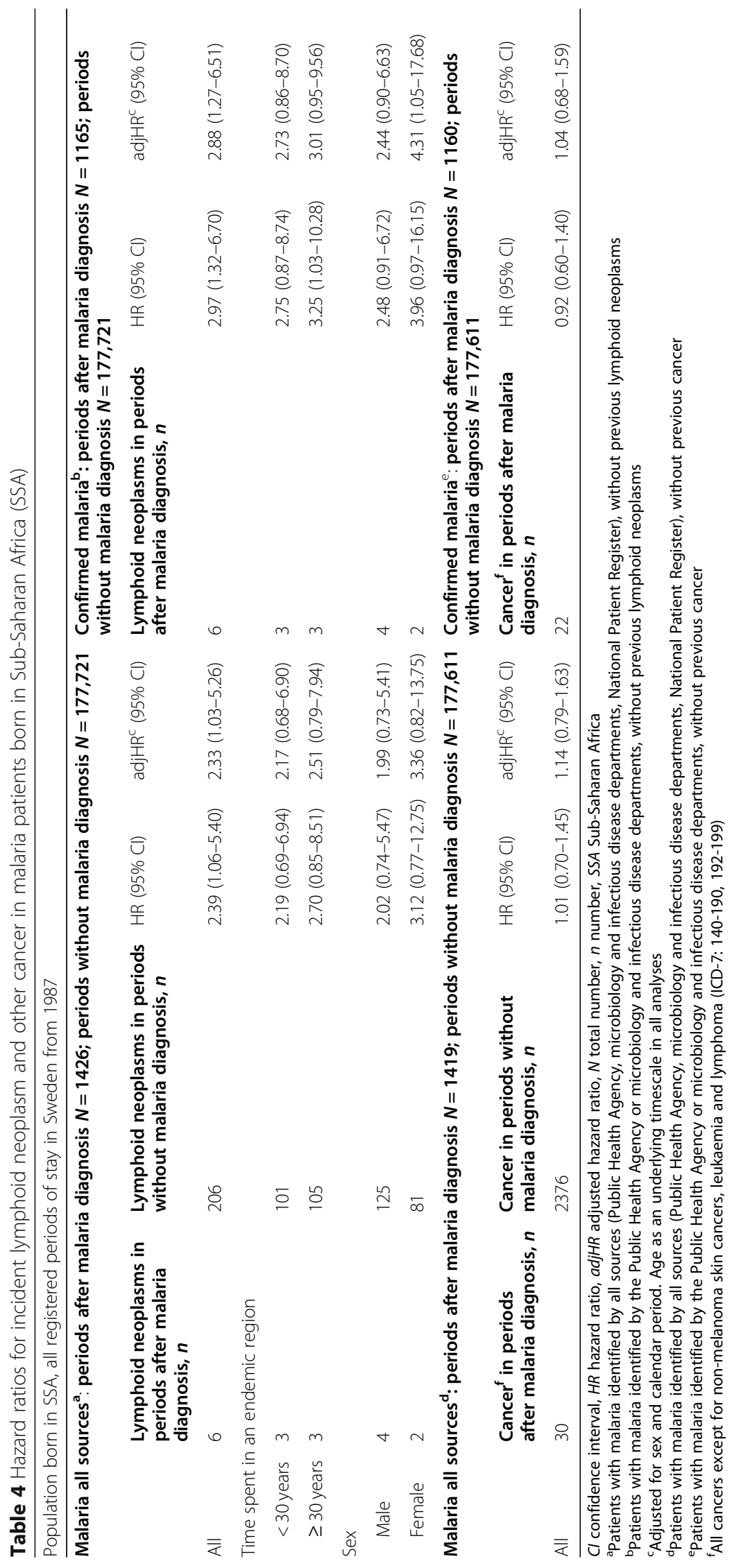


incidence and promote the growth of various lymphoid neoplasms however also involving an oncogenic virus [34, 35]. Assessment of EBV and other oncoviruses as co-factors was not possible in our cohort since such serological markers were not available.

In this study, a single episode of malaria in individuals from non-endemic regions was not associated with an elevated risk of lymphoid neoplasms. Neither could we observe any additional risk in patients with severe malaria, which in our setting mostly affects non-immune travellers with first-time malaria [21]. Analogous to studies of endemic Burkitt, repeated or chronic malaria exposure is a more probable requirement for lymphomagenesis [36, 37], and possibly this could explain why the association with lymphoma was observed in malaria patients that were born and had spent over 20 years in an endemic region. Moreover, within the population born in Sub-Saharan Africa, being diagnosed with malaria in Sweden might reflect a higher level of previous exposure.

Patients with malaria may differ from the general population with respect to socioeconomic status, education, tobacco and alcohol use. However, there is no evidence that these risk factors have an important role in lymphoma development [31, 38]. In addition, the risk of solid tumours, including the proportion of lung cancers, was lower among malaria patients from endemic regions; thus, it is unlikely that our results are substantially confounded by these risk factors.

Sex was included in adjusted analyses since NHL is more common among men [39], and also a larger proportion of malaria patients were male, but interestingly stratified analysis in the Sub-Saharan African cohort showed a tendency of increased risk among women with malaria.

Since HIV and chronic hepatitis B were diagnosed more often among malaria patients and both these, as well as hepatitis $C$, have been associated with lymphoma [40-42], we did an additional analysis excluding or censoring individuals with HIV and hepatitis before or during follow-up. This did not substantially affect the risk for lymphoid neoplasms in malaria patients with an endemic origin. In Sweden, HIV and hepatitis are included in the pre-treatment screening of patients with haematological malignancies [43], and since HIV and hepatitis diagnosed up to 6 months after lymphoma were censored, it is unlikely that any additional diagnoses would have been missed among individuals with lymphoma.

Some autoimmune diseases such as rheumatoid arthritis have also been associated to lymphoma [44], but information on previous comorbidities was often lacking in immigrants, and there is no evidence suggesting that inflammatory diseases would be more common among immigrants from malaria-endemic areas.

\section{Limitations and strengths}

This is to our knowledge the first published longitudinal study assessing the risk of incidental cancer in patients diagnosed with malaria. In addition to its nationwide population-based design with long-term follow-up, linkage with the Swedish national cancer register ensures unbiased and near-complete detection of the outcome. For confirmed malaria cases diagnosed from 1995, we also had clinical data enabling precise assessment of parasite species, disease severity and multiple episodes.

The setting in a malaria-free country where there is no risk of reinfection and malaria diagnoses are reported through compulsory notification aids correct classification of exposure.

However, misclassification of exposure, particularly among individuals with an endemic origin, cannot be entirely avoided since different degrees of exposure can have occurred before immigration or during later visits to endemic areas. Moreover, the persistence of undiagnosed asymptomatic infections after leaving the endemic setting can occur, as described in occasional case reports [45], but are likely to be rare. In addition, malaria transmission can vary greatly within a country as well as change over time, thus estimation of previous exposure based on country of birth will always be imprecise. We used the total number of years lived in an endemic region in an attempt to quantify previous exposure. However, even if the risk of lymphoma was somewhat increased in individuals with $>30$ years stay in endemic areas and confirmed malaria, data indicates that being diagnosed with malaria in Sweden might be a more adequate marker of high exposure.

The Swedish surveillance system for communicable diseases has been known for high sensitivity [46], but to ensure complete coverage, we also used the National Patient Register. Despite only including patients treated in hospitals and at outpatient clinics that manage malaria, there will still have been some degree of misdiagnosing, explaining the stronger association observed among confirmed cases.

The large proportion of immigrants resulted in partly incomplete follow-up due to frequent emigration, which we attempted to optimise by including subsequent reimmigration periods in the Sub-Saharan African cohort. This could potentially introduce a detection bias if patients diagnosed with malaria have a different migration pattern compared to the controls. Likewise, a presumably more active health care-seeking behaviour among immigrants diagnosed with malaria in Sweden would imply a higher probability of being diagnosed with other diseases such as lymphoma. However, both these potential biases would also have affected the incidence of allsite cancers, which we did not observe. Rather, there was a tendency of an overall lower cancer incidence in the Sub-Saharan African cohort, possibly due to selection of healthier individuals among immigrants (healthy migrant 
effect) or the return of migrants to their home country due to disease (salmon effect).

\section{Implications}

Our results indicate the need for a raised awareness among clinicians to consider lymphomas in malaria patients from endemic regions, many years after exposure. However, the study does not provide evidence for a causal link between malaria and lymphoid neoplasms, nor is it designed to understand the underlying mechanisms for the association observed. A larger cohort of malaria patients would be required to confirm our observations, preferably both with a similar setup in a non-endemic country and in regions of varying malaria endemicity.

\section{Conclusions}

A single episode of malaria in a traveller from a nonendemic country is not associated with an increased risk for lymphoid neoplasms. However, individuals that have grown up in Sub-Saharan Africa and diagnosed with malaria in Sweden have an increased risk for lymphoid neoplasms, in particular, B cell-derived lymphomas, several years after malaria diagnosis. There was no increased risk for non-haematological cancers in malaria patients independent of origin. The results suggest a possible association between repeated episodes of malaria during childhood and development of lymphoid neoplasm during adulthood.

\section{Supplementary information}

Supplementary information accompanies this paper at https://doi.org/10 1186/s12916-020-01759-8.

Additional file 1: Table S1. Crude and adjusted Hazard Ratio (HR) for incident lymphoid neoplasm and all-site cancer according to malaria exposure and region of birth. Table S2. Subtypes of lymphoid neoplasms in patients with malaria and comparators, according to region of birth. Table S3 Types of cancers (all) in patients with malaria and comparators, according to region of birth. Table S4. Crude and adjusted HR for incident lymphoid neoplasm, in population from Sub-Saharan Africa (SSA) without previous HIV and Chronic Hepatitis.

\section{Abbreviations}

ALL: Acute lymphoblastic leukaemia; Cl: Confidence interval; CLL: Chronic lymphatic leukaemia; EBV: Epstein-Barr virus; HR: Hazard ratio; adjHR: Adjusted hazard ratio; ICD: International Classification of Disease; InterLymph: International Lymphoma Epidemiology Consortium; NHL: NonHodgkin lymphoma; NPR: National Patient Register; PIN: Personal identification number; SNOMED: Systematized Nomenclature of Medicine; SSA: Sub-Saharan Africa; TPR: Total Population Register

\section{Acknowledgements}

We wish to thank Jonas Färnstrand at the Statistics of Sweden and Lars Holm at the National Board of Health and Welfare for the excellent advice throughout the register linkage process, as well as Marika Hjertquist and Elsie Ydring for contributing data from the Public Health Agency database. A special acknowledgement to Tobias Svensson and Ulf Hammar for their invaluable statistical support.

\section{Authors' contributions}

$A F, P N, K W, F G, M F$ and TD contributed to the design of the study. AW and $\mathrm{KW}$ collected the clinical data. KW analysed the data with assistance from FG.
All authors took part in the interpretation of the results. KW and AF drafted the initial manuscript. All authors have critically revised the manuscript for important intellectual content and approved the final version for submission. AF obtained the funding. The corresponding author attests that all listed authors meet the authorship criteria and that no others meeting the criteria have been omitted.

\section{Funding}

The study was funded by the Swedish Research Council, project grant 201802688, and Stockholm County Council, project grants 20150135 and 20180409

The researchers were independent from the funding source. The funders had no role in the design and conduct of the study; the collection, analysis and interpretation of the data; the preparation of the manuscript; or the decision to submit the manuscript for publication. Open access funding provided by Karolinska Institute.

\section{Availability of data and materials}

The data used in this study derives from the National Surveillance Database at the Public Health Agency of Sweden, the National Patient Register and the Cancer Register at the National Board of Health and Welfare in Sweden and the Total Population Register at Statistics of Sweden. The data contains sensitive personal information, and according to the Swedish law and the General Data Protection Regulation, the authors are not permitted to share the datasets used in this study with third parties. Details on the application procedures for data usage are available on the homepages of the respective registries. For statistical coding related to data analysis, contact the corresponding author at katja.wyss@sll.se.

\section{Ethics approval and consent to participate}

After linkage, national registration numbers were replaced by serial numbers through the Statistics of Sweden; thus, all analyses have been performed with anonymised data. The study was approved by the Ethical Review Board in Stockholm, Sweden (2009/1328-31/5, 2010/1080-32, 2012/1155-32, 2017/ 1006-32 and 2017/383-32)

\section{Consent for publication}

Not applicable

\section{Competing interests}

The authors declare that they have no competing interests.

\section{Author details}

'Division of Infectious Diseases, Department of Medicine Solna, Karolinska Institutet, Stockholm, Sweden. ${ }^{2}$ Department of Infectious Diseases, Karolinska University Hospital, 17176 Stockholm, Sweden. ${ }^{3}$ Clinical Epidemiology Division, Department of Medicine Solna, Karolinska Institutet, Stockholm, Sweden. ${ }^{4}$ Department of Infectious Diseases, Västerås Hospital, Västerås, Sweden. ${ }^{5}$ Division of Clinical Medicine, Department of Medicine Solna, Karolinska Institutet, Stockholm, Sweden. ${ }^{6}$ Function of Emergency Medicine, Karolinska University Hospital, Stockholm, Sweden.

Received: 17 June 2020 Accepted: 21 August 2020

Published online: 30 October 2020

\section{References}

1. World Health Organization. World malaria report 2019. Geneva: World Health Organization; 2019.

2. Hammerl L, Colombet M, Rochford R, Ogwang DM, Parkin DM. The burden of Burkitt lymphoma in Africa. Infectious Agents and Cancer. 2019;14:17.

3. Moormann AM, Snider CJ, Chelimo K. The company malaria keeps: how coinfection with Epstein-Barr virus leads to endemic Burkitt lymphoma. Curr Opin Infect Dis. 2011;24(5):435-41.

4. Bouvard V, Baan RA, Grosse Y, Lauby-Secretan B, El Ghissassi F, BenbrahimTallaa L, Guha N, Straif K, Canc WHOIAR. Carcinogenicity of malaria and of some polyomaviruses. Lancet Oncol. 2012;13(4):339-40.

5. Burkitt D. Malignant lymphoma in African children. Lancet (London, England). 1961;1(719):1410.

6. Kafuko GW, Burkitt DP. Burkitts lymphoma and malaria. Int J Cancer. 1970; $6(1): 1$. 
7. Rainey JJ, Omenah D, Sumba PO, Moormann AM, Rochford R, Wilson ML. Spatial clustering of endemic Burkitt's lymphoma in high-risk regions of Kenya. Int J Cancer. 2007;120(1):121-7.

8. Schmauz R, Mugerwa JW, Wright DH. The distribution of non-Burkitt, nonHodgkin's lymphomas in Uganda in relation to malarial endemicity. Int J Cancer. 1990;45(4):650-3.

9. Bates I, Bedu-Addo G. Chronic malaria and splenic lymphoma: clues to understanding lymphoma evolution. Leukemia. 1997;11(12):2162-7.

10. Quintana MDP, Smith-Togobo C, Moormann A, Hviid L. Endemic Burkitt lymphoma - an aggressive childhood cancer linked to Plasmodium falciparum exposure, but not to exposure to other malaria parasites. Apmis. 2020;128(2): 129-135.

11. Odida M, Schmauz R, Lwanga SK. Grade of malignancy of cervical cancer in regions of Uganda with varying malarial endemicity. Int J Cancer. 2002; 99(5):737-41.

12. Karimi P, Birmann BM, Anderson LA, McShane CM, Gadalla SM, Sampson JN, Mbulaiteye SM. Risk factors for Burkitt lymphoma: a nested case-control study in the UK Clinical Practice Research Datalink. Br J Haematol. 2018; 181(4):505-14.

13. Ross R, Dworsky R, Nichols P, Paganini-Hill A, Wright W, Koss M, Lukes R, Henderson B. Asbestos exposure and lymphomas of the gastrointestinal tract and oral cavity. Lancet (London, England). 1982;2(8308):1118-20.

14. Tavani A, La Vecchia C, Franceschi S, Serraino D, Carbone A. Medical history and risk of Hodgkin's and non-Hodgkin's lymphomas. Eur J Cancer Prev. 2000;9(1):59-64.

15. Vineis $P$, Crosignani $P$, Sacerdote C, Fontana A, Masala G, Miligi L, Nanni O, Ramazzotti V, Rodella S, Stagnaro E, et al. Haematopoietic cancer and medical history: a multicentre case control study. J Epidemiol Community Health. 2000;54(6):431-6.

16. Chen CJ, Liang KY, Chang YS, Wang YF, Hsieh T, Hsu MM, Chen JY, Liu MY Multiple risk-factors of nasopharyngeal carcinoma - Epstein-Barr virus, malarial infection, cigarette-smoking and familial tendency. Anticancer Research. 1990;10(2B):547-53.

17. Ludvigsson JF, Otterblad-Olausson P, Pettersson BU, Ekbom A. The Swedish personal identity number: possibilities and pitfalls in healthcare and medical research. Eur J Epidemiol. 2009;24(11):659-67.

18. Ludvigsson JF, Andersson E, Ekbom A, et al. External review and validation of the Swedish National Inpatient Register. BMC Public Health. 2011;11(450). https://doi.org/10.1186/1471-2458-11-450.

19. Farnert A, Wyss K, Dashti S, Naucler P. Duration of residency in a nonendemic area and risk of severe malaria in African immigrants. Clin Microbiol Infection. 2015;21(5):494-501.

20. Wyss K, Wangdahl A, Vesterlund M, Hammar U, Dashti S, Naucler P, Farnert A. Obesity and diabetes as risk factors for severe Plasmodium falciparum malaria: results from a Swedish Nationwide Study. Clin Infect Dis. 2017;65(6):949-58.

21. Wangdahl A, Wyss K, Saduddin D, Bottai M, Ydring E, Vikerfors T, Farnert A Severity of Plasmodium falciparum and non-falciparum malaria in travelers and migrants: a nationwide observational study over 2 decades in Sweden. J Infect Dis. 2019:220(8):1335-45.

22. Division UNS. Standard country or area codes for statistical use (M49). Available from: https://unstats.un.org/unsd/methodology/m49/ Accessed 2019-12-10

23. Barlow L, Westergren $K$, Holmberg $L$, Talback M. The completeness of the Swedish Cancer Register - a sample survey for year 1998. Acta Oncol. 2009; 48(1):27-33.

24. Morton LM, Turner JJ, Cerhan JR, Linet MS, Treseler PA, Clarke CA, Jack A, Cozen W, Maynadie M, Spinelli JJ, et al. Proposed classification of lymphoid neoplasms for epidemiologic research from the Pathology Working Group of the International Lymphoma Epidemiology Consortium (InterLymph). Blood. 2007;1 10(2):695-708.

25. Turner JJ, Morton LM, Linet MS, Clarke CA, Kadin ME, Vajdic CM, Monnereau A, Maynadie M, Chiu BCH, Marcos-Gragera R, et al. InterLymph hierarchical classification of lymphoid neoplasms for epidemiologic research based on the WHO classification (2008): update and future directions. Blood. 2010; 116(20):E90-8

26. Carpenter LM, Newton R, Casabonne D, Ziegler J, Mbulaiteye S, Mbidde E, Wabinga $\mathrm{H}$, Jaffe $\mathrm{H}$, Beral V. Antibodies against malaria and Epstein-Barr virus in childhood Burkitt lymphoma: a case-control study in Uganda. Int Cancer. 2008;122(6):1319-23.

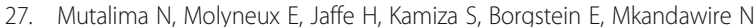
Liomba G, Batumba M, Lagos D, Gratrix F et al: Associations between Burkitt lymphoma among children in Malawi and infection with HIV, EBV and malaria: results from a case-control study. PLoS One 2008;3(6). https://doi. org/10.1371/journal.pone.0002505.

28. Legason ID, Pfeiffer RM, Udquim KI, Bergen AW, Gouveia MH, Kirimunda S, Otim I, Karlins E, Kerchan P, Nabalende H, et al. Evaluating the causal link between malaria infection and endemic Burkitt lymphoma in Northern Uganda: a Mendelian randomization study. Ebiomedicine. 2017;25:58-65.

29. Mwanda OW, Rochford R, Moormann AM, Macneil A, Whalen C, Wilson ML: Burkitt's lymphoma in Kenya: geographical, age, gender and ethnic distribution. East Afr Med J 2004(8 Suppl):S68-77.

30. de Martel C, Ferlay J, Franceschi S, Vignat J, Bray F, Forman D, Plummer M. Global burden of cancers attributable to infections in 2008: a review and synthetic analysis. Lancet Oncol. 2012;13(6):607-15.

31. Smedby KE, Ponzoni M. The aetiology of B-cell lymphoid malignancies with a focus on chronic inflammation and infections. J Intern Med. 2017;282(5): 360-70.

32. Thorley-Lawson D, Deitsch KW, Duca KA, Torgbor C: The link between Plasmodium falciparum malaria and endemic Burkitt's lymphoma-new insight into a 50-year-old enigma. PLoS Pathog 2016;12(1). https://doi.org/ 10.1371/journal.ppat.1005331.

33. Torgbor C, Awuah P, Deitsch K, Kalantari P, Duca KA, Thorley-Lawson DA. A multifactorial role for $P$. falciparum malaria in endemic Burkitt's lymphoma pathogenesis. PLoS Pathogens. 2014;10(5):e1004170.

34. Wedderburn N: Effect of concurrent malarial infection on development of virus-induced lymphoma in balb/C MICE. Lancet (London, England) 1970, 2(7683):1114.

35. Jerusalem C. Relationship between malaria infection (Plasmodium berghei) and malignant lymphoma in mice. Zeitschrift Fur Tropenmedizin Und Parasitologie. 1968;19(1):94-108.

36. Moormann AM, Bailey JA. Malaria - how this parasitic infection aids and abets EBV-associated Burkitt lymphomagenesis. Current Opinion in Virology. 2016;20:78-84.

37. Johnston WT, Mutalima N, Sun D, Emmanuel B, Bhatia K, Aka P, Wu X Borgstein E, Liomba GN, Kamiza S, et al. Relationship between Plasmodium falciparum malaria prevalence, genetic diversity and endemic Burkitt lymphoma in Malawi. Sci Rep. 2014;4:3741.

38. Morton LM, Hartge P, Holford TR, Holly EA, Chiu BC, Vineis P, Stagnaro E, Willett EV, Franceschi S, La Vecchia C, et al. Cigarette smoking and risk of non-Hodgkin lymphoma: a pooled analysis from the International Lymphoma Epidemiology Consortium (InterLymph). Cancer Epidemio Biomark Prev. 2005;14(4):925-33.

39. Szekely E, Hagberg $O$, Arnljots K, Jerkeman M. Improvement in survival of diffuse large B-cell lymphoma in relation to age, gender, International Prognostic Index and extranodal presentation: a population based Swedish Lymphoma Registry study. Leuk Lymphoma. 2014;55(8):1838-43.

40. Dalia S, Chavez J, Castillo JJ, Sokol L. Hepatitis B infection increases the risk of non-Hodgkin lymphoma: a meta-analysis of observational studies. Leuk Res. 2013;37(9):1107-1115. https://doi.org/10.1016/j.leukres.2013.06.007.

41. van Leeuwen MT, Vajdic CM, Middleton MG, McDonald AM, Law M, Kaldor JM, Grulich AE. Continuing declines in some but not all HIV-associated cancers in Australia after widespread use of antiretroviral therapy. Aids. 2009;23(16):2183-90.

42. Su TH, Liu CJ, Tseng TC, Chou SW, Liu CH, Yang HC, Wu SJ, Chen PJ, Chen DS, Chen $\mathrm{CL}$, et al. Hepatitis $\mathrm{C}$ viral infection increases the risk of lymphoidneoplasms: a population-based cohort study. Hepatology. 2016;63(3):721-30.

43. Regionala cancercentrum i samverkan. Aggressiva B-cellslymfom, Nationellt Vårdprogram (Swedish regional cancercenters in cooperation. Aggressive Bcell lymphoma, national guidelines), Version 4.0, Sept 2019.

44. Hellgren K, Baecklund E, Backlin C, Sundstrom C, Smedby KE, Askling J. Rheumatoid arthritis and risk of malignant lymphoma. Arthritis Rheumatol. 2017;69(4):700-8.

45. Ashley EA, White NJ. The duration of Plasmodium falciparum infections. Malar J. 2014;13. https://doi.org/10.1186/1475-2875-13-500.

46. Jansson A, Arneborn M, Ekdahl K. Sensitivity of the Swedish statutory surveillance system for communicable diseases 1998-2002, assessed by the capture-recapture method. Epidemiol Infect. 2005;133(3):401-7.

\section{Publisher's Note}

Springer Nature remains neutral with regard to jurisdictional claims in published maps and institutional affiliations. 